\title{
Development of the safety code AINA for the European DEMO designs
}

\author{
Eduard Baeza ${ }^{\mathrm{a}}$, Alfredo de Blas ${ }^{\mathrm{a}}$, Albert Riego ${ }^{\mathrm{a}}$, Marco Fabbri ${ }^{\mathrm{a}}$ \\ ${ }^{a}$ Fusion Energy Engineering Laboratory, Technical University of Catalonia (UPC) Barcelona-Tech, Barcelona, Spain
}

\begin{abstract}
In order to evaluate plasma evolution and in-vessel components strains, a safety code called AINA has been developing during the last ten years for different fusion reactors designs. This work describes the new AINA code which is being adapted for the four European DEMO designs (HCPB, DCLL, HCLL and WCLL) after an in-depth critical analysis of the former AINA versions with the purpose of performing a proper, reliable, versatile and flexible tool for the future safety studies. At this point, a new 0D plasma dynamics approach and a 1D finitedifference thermal model for the DEMO HCPB configuration and the divertor have been developed. By means of the feedback among both blocks, a preliminary safety analysis is carrying out checking the integrity of in-vessel components both when a plasma perturbation induces a Loss Of Plasma Control (LOPC) and a thermo-hydraulic accident takes place in the Plasma Facing Components (PFCs) or in the Vacuum Vessel such as a Loos Of Coolant Accident (LOCA).

Initial results show deficiencies in the Blanket design which may be extremely significant when some of the described unexpected scenarios takes place leading the reactor to a melting episode.
\end{abstract}

Keywords: safety, DEMO, AINA, plasma physics, thermal analysis, LOCA.

\section{Introduction}

A conclusion that can be drawn from the historical safety analyses developed for tokamaks fusion reactors is that some of the major risks involve incidents in the vacuum vessel. In order to evaluate plasma evolution and in vessel components strains, a safety code called AINA (acronym of Analyses of IN-vessel Accidents) has been developing by the Fusion Energy Engineering Laboratory (FEEL) of the Technical University of Catalonia (UPC) Barcelona-Tech, during the last ten years for different fusion reactors designs as ITER [1-5] and the Japanese DEMO design WCPB [6].

An in-depth critical analysis of the former AINA versions, a new codification and a checking and validation phase have been performed in order to develop a proper, reliable, versatile and flexible tool with the purpose of carrying out safety analyses for the four European DEMO designs (HCPB, DCLL, HCLL and WCLL).

At this point, AINA is a code comprised of a $0 \mathrm{D}$ plasma dynamics approach based on a mass and energy balance and a 1D thermal model for the blanket (in the radial direction), specifically for the HCPB configuration, and the divertor. These two blocks feedback constantly each other by means of the plasma-wall block which estimates the real loads suffered by the in vessel components and the real impurity presence into the plasma core. With this basic concept, AINA is useful to check the integrity of these in-vessel components both when a plasma perturbation induces a Loss Of Plasma Control (LOPC) and a thermo-hydraulic accident takes place in the Plasma Facing Components (PFCs) or in the Vacuum Vessel such as a Loos Of Coolant Accident (LOCA). This document describes the new AINA code, specifically the models and the numerical procedures implemented in each block.

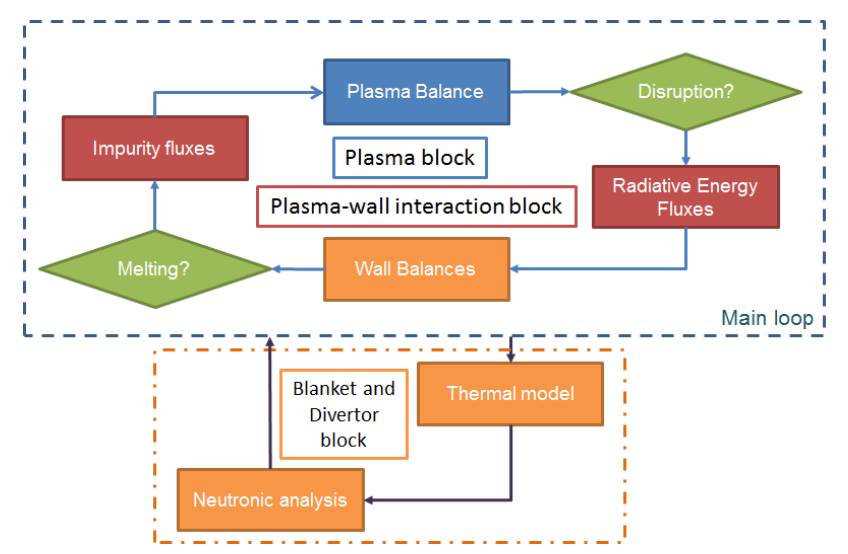

Fig. 1. AINA scheme.

\section{Plasma block}

\subsection{Equations}

As stated before, the code considers a 0D multi-fluid approach based on the mass and energy balance of the plasma core according to the equations showed below. Particle conservation is considered for fuel ions $\left(n_{H}\right)$ alpha particles $\left(n_{\alpha}\right)$ and every type of impurity $\left(n_{Z X e}\right.$ and $n_{X W}$ which are referred to Xenon and Tungsten). On the other hand, the energy conservation expressions considered treat ions and electrons separately. It is important to highlight that all the terms are calculated through volume and radial profiles of plasma density and temperature using the same models as AINA 3.0 [3]. AINA determines a SS (steady state) scenario of the plasma using an average ion temperature and a specified power fusion as inputs and solving the system by the Newton method. 


$$
\begin{aligned}
& \frac{d n_{H}}{d t}=S_{H}-2 S_{\alpha}-\frac{n_{H}}{\tau_{p, H}} \\
& \frac{d n_{\alpha}}{d t}=S_{\alpha}-\frac{n_{\alpha}}{\tau_{p, \alpha}} \\
& \frac{d n_{Z X e}}{d t}=S_{Z X e}-\frac{n_{Z X e}}{\tau_{p, Z}} \\
& \frac{d n_{Z W}}{d t}=S_{Z W}-\frac{n_{Z W}}{\tau_{p, Z}} \\
& \frac{3}{2} \frac{d\left(n_{i} T_{i}\right)}{d t}=f_{e x t} P_{e x t}+f_{\alpha} P_{\alpha}-P_{i e}-\frac{3}{2} \frac{n_{i} T_{i}}{\tau_{E, i}} \\
& \frac{3}{2} \frac{d\left(n_{e} T_{e}\right)}{d t}=\left(1-f_{e x t}\right) P_{e x t}+\left(1-f_{\alpha}\right) P_{\alpha} \\
& +P_{i e}+P_{O h m}-P_{B r}-P_{S y}-P_{l i}-\frac{3}{2} \frac{n_{e} T_{e}}{\tau_{E, e}} \\
& n_{e}=n_{i}+2 n_{\alpha}+\sum_{j=1}^{\infty}\left(Z_{j} n_{Z j}\right)
\end{aligned}
$$

The initial condition for the first iteration considers an external power $\left(P_{\text {ext }}\right)$ and the alpha source and the hydrogen density estimation via the next expressions:

$$
S_{\alpha}=\frac{p_{f u s}}{E_{f u s}} ; \quad n_{H}=\sqrt{\frac{4 S_{\alpha}}{\langle\sigma v\rangle_{D T} \cdot V}}
$$

Where $E_{f u s}=17.62 \mathrm{MeV}$ and $V$ is the plasma volume. When the SS parameters are calculated, the time evolution of the plasma is estimated by the Euler method. The rest of the components present in the balance such as the velocity averaged cross-section of the D-T nuclear fusion reaction or the radiation powers as well as the plasma equilibrium limits or the scaling laws for the confinement energy time have been properly modeled, referenced and discussed in [7].

\section{Thermal blanket block}

The breeding blanket is one of the most challenging and innovative components due to the high strains it suffers. Moreover, several cooling loops embedded inside this component are responsible for maintaining the temperature within reasonable regimes and extracting the undesirable tritium in excess. The determination of 3D detailed temperature distribution by means of analytic method is not feasible thus it requires the usage of Computational Fluid Dynamics (CFD), as ANSYS FLUENTC [8] which are very demanding from a computational point of view; and this matter is not be consistent with the AINA approach about fast processing. For these reasons, flexible thermalhydraulics routines, based on the finite differences technique, have been developed in order to obtain reliable, approximate and conservative (in comparison with the 3D model) 1D, radial and time-dependent simplified thermal-wall model in a short notice using a standard workstation [9]. In addition, these routines must take into consideration the influence of coolant channels not in line with the 1D segment which are present in the European DEMO designs. The effect of these tubes is considered using a weighted convective negative flux effect in function of the radial distance from the coolant and the poloidal distance from the 1D discretization line. The final expressions modeled are:

$$
\begin{aligned}
& \rho(T, z) c(T, x) \frac{\partial T(x, t)}{\partial t}=\frac{\partial}{\partial x}\left(\begin{array}{l}
\left(1-f_{\text {COOL }_{j}}\right) k_{T}(T, x) \frac{\partial T(x, t)}{\partial x} \\
+f_{\text {COOL }_{j}} h_{j}\left(T(x, t)-T_{j, \infty}(x, t)\right)
\end{array}\right) \\
& +f_{W G T, R} f_{W G T, P} h_{k}\left(T(x, t)-T_{k, \infty}(x, t)\right)+\dddot{q}(x, t) \\
& f_{\text {COOL }_{j}}(x)=\sum \partial_{j}(x) \frac{\text { Surface }_{\text {cooled }}}{\text { Surface }_{\text {Total }}} \\
& f_{\text {WGT }, R}(x)=\sum \partial_{k}(x) f_{W G T, R} \\
& f_{W G T, P}(x)=\sum \partial_{k}(x) f_{W G T, P}
\end{aligned}
$$

Where $\rho$ is the material density, $c$ the heat capacity, $k_{T}$ the thermal conductivity, $\dddot{q}$ the volumetric nuclear heat deposition, $h$ is the heat transfer coefficient, $T(x)$ the material temperature at $x, T_{i, \infty}$ the coolant bulk temperature for the tube $i, f_{\text {cool }}$ the coolant surface for the in line tube which is equal to the relative surface of the coolant tubes to the total surface of the module section, $f_{W G T, R}$ and $f_{W G T, P}$ the coolant factor for the no in lines tubes that are discrete functions which take values only at the specified coolant positions and $\delta$ is the Dirac delta function.

The blanket region modeled is OB4 due to it is usually one that suffers the maximum load [10]. The equation is solved considering the total load due to the radiation effect $\left(P_{\mathrm{rad}}\right)$ in the first node estimated by the plasma block, a Robin boundary condition for the last node by means of a heat transfer coefficient and a bulk temperature condition at the back side of the blanket and a nuclear heating distribution scaled for the Neutron Wall Load $(N W L)$ estimated by the plasma block.

The material properties, the model discretization, the nuclear heating distribution and the boundary conditions associated to the cooling system depend on the blanket design modeled, currently, the HCPB latest version (HCPB-2015 v3 [10]) has been selected.

A $2 \mathrm{~mm} \mathrm{~W}$-armour layer is assumed for all the modules at the plasma facing side of the FW whereas in the internal part, the Cooling Plate subdivides the Be and the $\mathrm{LiSiO}_{4}$ bed zone which are arranged perpendicularly to the FW and alternated. The resulting 1D HCPB AINA thermal blanket model assumed is composed of layers properly discretized defining the No. of nodes for each material slab. After a pertinent analysis, the Tungsten FW is divided into 10 nodes, the coolant layers are represented by a single node and the rest are discretized into 500 nodes.

Thanks to the AINA Wall thermal model flexibility the layer No.5 can compute the Be, the $\mathrm{LiSiO}_{4}$ and the EUROFER temperature profile.

Besides the FW cooling, the HCPB cooling system is provided by two Helium redundant, fully symmetric, purely counter flow, coolant scheme which each one provide $50 \%$ of the cooling performance. All pipes have been duly modeled via the numerical expression already exposed ( $\underline{3})$. 


\section{Thermal divertor block}

The divertor as main interface component between the plasma and the components material, it shall tolerate high heat loads, for this reason a first divertor model has been implemented in AINA.

Both the numerical model and the solver and all the requirements (boundary conditions, material properties...) are the same as used in the thermal blanket block although they are adapted to the divertor configuration. Moreover, the dimensional segment modeled is the most demanded which crosses along the Outer Vertical Target and the Cassette Body.

Following a discussed study, the resulting 1D AINA thermal divertor model is composed by the pertinent layers discretized into 500 nodes for the no coolant slabs and a single node for the coolant slabs.

\section{Plasma wall interaction block}

This block is responsible for interconnecting the plasma with the thermal blocks and is focused on:

- Estimation of the loads $\left(P_{r a d}\right.$ and $\left.N W L\right)$ for the thermal equilibrium calculation.

- Estimation of the impurity fluxes to the plasma core for the plasma mass and energy balance.

\subsection{Wall loads}

The neutron wall load is used to scale the nuclear heating distribution in the thermal blocks. Its average value is calculated as:

$$
N W L_{\text {Ave }}=\frac{S_{\alpha} \cdot\langle\sigma v\rangle_{D T} \cdot E_{n}}{S_{\text {wall }}} \cdot F_{\text {mult }}
$$

Where $E_{n}$ is the mean energy of neutrons, $S_{\text {wall }}$ is the surface of the wall (FW and divertor) and $F_{\text {mult }}$ is a multiplication factor embedded due to the incident $N W L$ power will be "multiplied" in the Breeder Blankets [10]. The NWL distribution for every module of the wall is derived from the Table 4.6 of the document by Hernandez et a [10].

On the other hand, the radiative heat flux is used as a boundary condition in the first node of the thermal blocks. Its total value is estimated as [11]:

$$
P_{\text {rad }}=R W L+R D L-\dot{q}_{\text {Rad } \operatorname{Re} f l}-\dot{q}_{\text {eros }}
$$

The $R W L_{\text {Ave }}$, is the average radiation load on the first wall its distribution for every module of the wall is derived from the Table 5.2 of the document by Hernandez et a [10]; and the $R D L$ is the radiation load on the divertor.

$$
\begin{aligned}
& R W L_{\text {Ave }}=\frac{P_{B r}+P_{s y}+P_{l i}+P_{\text {edge }}}{S_{\text {wall }}}+\frac{P_{\alpha} \cdot 2 \cdot f_{\text {ripple }}}{S_{\text {wall }}} \\
& R D L=\frac{P_{\text {SOL }}-P_{\text {edge }}-P_{\text {Div_rad }}}{S_{\text {wall }}} \\
& P_{S O L}=P_{\text {ext }}+P_{\alpha}-P_{B r}-P_{s y}-P_{l i}
\end{aligned}
$$

And $P_{D i v \text { rad }}$ is the radiation from the divertor region and it is estimated using an accepted lineal regression [12].

The rest of the components of the expression (5) are the radiation reflected for every module of the blanket, and the flux emitted due to impurity flux leaving the wall. They have not been implemented yet, thus assuming a more conservative calculation.

\subsection{Impurities}

The presence of $\mathrm{Xe}$ and $\mathrm{W}$ inside the plasma core is governed by the following assumptions:

- Regarding Xenon: a constant fraction of Xenon is desired inside the plasma core $(0.0389 \%$ of the electron density).

- Regarding Tungsten: its production model is composed by two main sources: thermal sublimation and physical sputtering.

The thermal sublimation source is calculated as a function of the PFC temperature by means of the following model exposed by Uckan [13]:

$$
\Gamma_{\text {Subli }_{i}}=\frac{2.6 \cdot 10^{14}}{\sqrt{M_{i} \cdot T}} \cdot 10^{\frac{B_{i}-A_{i}}{T}}
$$

Where $T$ is the PFC surface temperature, $M_{i}$ is the atomic mass of the PFC material, $A_{i}$ and $B_{i}$ are fixed coefficients.

The physical sputtering model used in AINA is based on empirical formulas where the sputtering yield is described as a function of the projectile energy $E_{0}$ at normal incidence which was formulated by Bohdansky [14] and improved by Wilson $[15,16]$ :

$$
Y_{\text {Phys Sout }}\left(E_{0}\right)=Q \cdot S_{n}(\varepsilon) \cdot\left(1-\left(\frac{E_{\text {th }}}{E_{0}}\right)^{2 / 3}\right) \cdot\left(1-\frac{E_{\text {th }}}{E_{0}}\right)^{2}
$$

Ion fluxes, neutral particle fluxes and their corresponding energies on the surface of the PFCs necessaries to calculate the erosion on it has been estimated via a discussed extrapolation and scaling process from the results of an ITER simulation [17] and a multi-machine comparison [18].

On the other hand, a time delay of transport should be considered since the impurities do not reach the plasma core instantly. It is known that a laser ablation experiment [19-21] shows a fast transport of impurities into the plasma. Therefore, a time delay of one energy confinement time could be judged as conservative from a safety point of view [22]

$$
S_{Z_{i}}(t)=C_{\text {screen }_{Z_{i}}} \cdot\left(t-\tau_{E, e}\right)
$$

Where $S_{Z i}$ is the $i$ impurity source and $C_{\text {screenzi }}$ is the screening factor for the impurity $i$ calculated during the steady state estimation and remaining for transients. 


\section{Simulations}

\subsection{Steady State scenario}

AINA 4.0 steady state simulation of DEMO1 scenario [23] is presented in the next table:

Table 1. Main global parameters of the DEMO1 computed by means of AINA 4.0 and compared with PROCESS results.

\begin{tabular}{|c|c|c|}
\hline INPUTS & \multicolumn{2}{|c|}{ DEMO1 } \\
\hline Major radius [m] & \multicolumn{2}{|c|}{9.072} \\
\hline Minor radius [m] & \multicolumn{2}{|c|}{2.927} \\
\hline Toroidal field $[\mathrm{T}]$ & \multicolumn{2}{|c|}{5.667} \\
\hline Safety factor $95 \%$ flux & \multicolumn{2}{|c|}{3.247} \\
\hline Plasma volume $\left[\mathrm{m}^{3}\right]$ & \multicolumn{2}{|c|}{2502} \\
\hline Plasma surface [m²] & \multicolumn{2}{|c|}{1428} \\
\hline Fusion power [MW] & \multicolumn{2}{|c|}{2037} \\
\hline Ion temperature $[\mathrm{keV}]$ & \multicolumn{2}{|c|}{13.065} \\
\hline OUTPUTS & AINA & PROCESS \\
\hline Electron temp. [keV] & 13.04 & 13.065 \\
\hline Fuel source $\left[\mathrm{m}^{-3} \mathrm{~s}^{-1}\right]$ & $8.95 \mathrm{e} 18$ & $2.82 \mathrm{e} 18$ \\
\hline Electron density $\left[\mathrm{m}^{-3}\right]$ & $8.35 \mathrm{e} 19$ & $7.98 \mathrm{e} 19$ \\
\hline Ion density $\left[\mathrm{m}^{-3}\right]$ & $7.55 \mathrm{e} 19$ & $6.99 \mathrm{e} 19$ \\
\hline Hydrogen density $\left[\mathrm{m}^{-3}\right]$ & $6.94 \mathrm{e} 19$ & $6.144 \mathrm{e} 19$ \\
\hline Alpha density $\left[\mathrm{m}^{-3}\right]$ & $5.98 \mathrm{e} 18$ & $7.98 \mathrm{e} 18$ \\
\hline Impurity density $\left[\mathrm{m}^{-3}\right]$ & $3.66 \mathrm{e} 16$ & $3.51 \mathrm{e} 16$ \\
\hline $\mathrm{Xe}$ density $\left[\mathrm{m}^{-3}\right]$ & $3.25 \mathrm{e} 16$ & - \\
\hline $\mathrm{W}$ density $\left[\mathrm{m}^{-3}\right]$ & $4.17 \mathrm{e} 15$ & - \\
\hline Xe fraction $[\%]$ & 0.0389 & 0.0389 \\
\hline W fraction $[\%]$ & 0.005 & 0.005 \\
\hline External power [MW] & 66.4 & 50 \\
\hline Gain & 30.68 & 39.86 \\
\hline Alpha Power [MW] & 399 & 407 \\
\hline Ion-Elec.exchange [MW] & 1.76 & 0 \\
\hline Ohmic power [MW] & 0.95 & 1.1 \\
\hline Bremss. power [MW] & 80.98 & 87.9 \\
\hline Synchr. power [MW] & 30.2 & 25.9 \\
\hline Line power [MW] & 226.3 & 191 \\
\hline Edge power $[\mathrm{MW}]$ & 172 & 172.9 \\
\hline Radiation in core [MW] & 165.6 & 132.6 \\
\hline Total Radiation [MW] & 337.6 & 305.5 \\
\hline SOL power $[\mathrm{MW}]$ & 300 & - \\
\hline Beta total $[\%]$ & 3.26 & 3.1 \\
\hline Beta toroidal [\%] & 2.81 & 3.2 \\
\hline Beta poloidal [\%] & 0.96 & 1.1 \\
\hline Confinement time [s] & 4.15 & 4.23 \\
\hline Plasma current [MA] & 20.3 & 19.6 \\
\hline Bootstrap fraction $[\%]$ & 0.25 & 0.32 \\
\hline $\mathrm{NWL}_{\text {Ave }}\left[\mathrm{MW} / \mathrm{m}^{2}\right]$ & 1.07 & 1.05 \\
\hline $\mathrm{P}_{\mathrm{rad}}\left[\mathrm{MW} / \mathrm{m}^{2}\right]$ & 0.17 & 0.22 \\
\hline
\end{tabular}

These values obtained from the AINA simulation are similar to those obtained from PROCESS and no meaningful discrepancies have been found.

Notwithstanding, it is necessary to highlight that for the DEMO1 scenario certain functional temperature limits (EUROFER, Beryllium and $\mathrm{LiSiO}_{4}$ ) for the HCPB $\mathrm{BB}$ design are slightly exceeded in the worst poloidal region as envisaged by thermo-hydraulic analyses $[7,10,24]$ and as exposed in table 2 .

Accordingly, it would be advisable to undertake a design review focused on ensuring a suitable operating temperature range for all the materials which make up the HCPB blanket.
Table 2. HCPB AINA DEMO1 SS maximum temperature.

\begin{tabular}{llcc}
\hline Region & Material & $\mathrm{T}$ limit $\left[{ }^{\circ} \mathrm{C}\right]$ & $\mathrm{T} \mathrm{max}\left[{ }^{\circ} \mathrm{C}\right]$ \\
\hline $\mathrm{BB} / \mathrm{Div}$ & Tungsten & 3422 & $505 / 182$ \\
$\mathrm{BB} / \mathrm{Div}$ & EUROFER & 550 & $578 / 317$ \\
$\mathrm{BB}$ & $\mathrm{Be}$ & 650 & 816 \\
$\mathrm{BB}$ & $\mathrm{LiSiO}_{4}$ & 920 & 1360 \\
Div & $\mathrm{Cu}$ & 980 & 158 \\
Div & $\mathrm{CuCrZr}$ & 1050 & 156 \\
\hline
\end{tabular}

\subsection{Transients evolution}

The vast majority of the Postulated Initial Events assumed in DEMO [25] may induce the following load or accident scenarios which AINA is able to simulate:

- Plasma disruption or structural material melting due to a LOPC.

- In-vessel melt either of FW, blanket structure and/or divertor regions because of thermal stresses due to a LOCA.

In future, a detailed safety study will be carry out and several perturbations which may affect the reactor integrity will be analyzed. At this point, it has been noted that some perturbations such as an external power cutoff, an increase of $25 \%$ in the fuel injection or a LOCA could lead to a worsen scenario from the temperature limits point of view, whilst a fueling injection cut-off or a fueling rate increase above $25 \%$ could induce plasma disruptions with very high thermal energies.

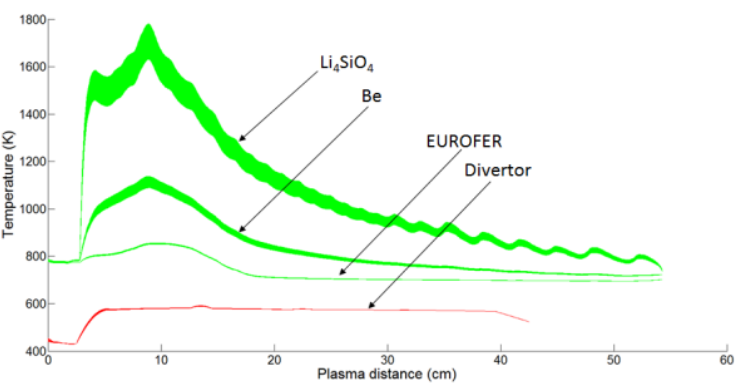

Fig. 2. Increase of the $\mathrm{BB}$ and divertor temperatures after an external power cut-off.

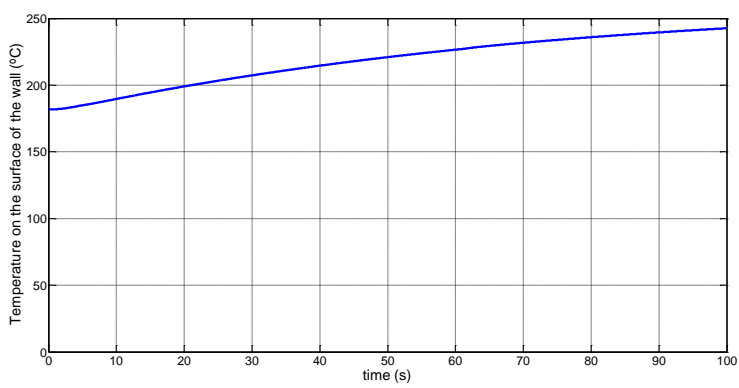

Fig. 3. Surface divertor temperature after a loss of $90 \%$ of the mass flow rate in the PFC loop.

\section{Conclusions}

The new AINA has become a proper, reliable, versatile and flexible tool in order to preform future safety studies for the European DEMO designs. 
Several potential risk scenarios as LOPCs and LOCAs can be simulated thus the most critical will be identified.

It would be advisable to undertake a design review focused on ensuring a suitable operating temperature range for all the materials which make up the HCPB.

\section{Future Work}

On the basis of this previous work, future tasks will be focused on the development of the HCPB safety analysis by means of the new AINA, the adaptation of the thermal blanket block for the rest of the European blanket designs (DCLL, HCLL and WCLL) and their pertinent safety analyses.

\section{References}

[1] J. Dies, M. Dapena, M. Ramon, J. Garcia, J.C. Rivas, A. Calvo, S. Reyes, AINA safety code, a review of loss of plasma control transients in ITER: sudden increase in fueling rate, sudden increase of auxiliary heating, Fusion Science and Technology. 56 (2009) 31-37.

[2] J.C. Rivas, J. Dies, Upgrading of plasma wall interaction model for tokamak transient modeling code AINA 2.0, used in safety studies of ITER plasma instability events, Fusion Science and Technology. 60 (2011) 825-829.

[3] J.C. Rivas, J. Dies, Safety studies: Review of loss of plasma control transients in ITER with AINA 3.0 code, Fusion Engineering and Design. 88 (2013) 2709-2713. http://dx.doi.org/10.1016/j.fusengdes.2013.02.134

[4] J.C. Rivas, J. Dies, ITER safety studies: The effect of two simultaneous perturbations during a loss of plasma control transient, Fusion Engineering and Design. 89 (2014) 2043-2047. http://dx.doi.org/10.1016/j.fusengdes.2014.04.010.

[5] J.C. Rivas, J. Dies, X. Fajarnés, Revisiting the analysis of passive plasma shutdown during an ex-vessel loss of coolant accident in ITER blanket, Fusion Engineering and Design. 98-99 (2015) 2206-2209.

http://dx.doi.org/10.1016/j.fusengdes.2015.06.143

[6] J.C. Rivas, M. Nakamura, Y. Someya, K. Hoshino, N. Asakura, H. Takase, Y. Miyoshi, H. Utoh, K. Tobita, J. Dies, A. de Blas, A. Riego, M. Fabbri, Safety studies of plasma-wall events with AINA code for Japanese DEMO, Fusion Engineering and Design. (2015). https://dx.doi.org/10.1016/j.fusengdes.2015.10.037

[7] A. De Blas, A. Riego, E. Baeza, M. Fabbri, Á. Cubí, Report on development of new AINA code for DEMO HCPB (models and numerical methods), (EUROfusion document, Working Package of Safety and Environment).

[8] ANSYS Team, ANSYS Fluent (C) User Manual, 2015.

[9] M. Fabbri, A. de Blas, A. Riego, I. Zamora, E. Baeza, Methodology for the improvement of the AINA Code wall-model applied to DEMO WCPB blanket, Fusion Engineering and Design, ISSN 0920-3796, (2017). http://dx.doi.org/10.1016/j.fusengdes.2017.05.027

[10] F.A. Hernández, Q. Kang, B. Kiss, P. Norajitra, G. Nádasi, P. Pereslavtsev, C. Zeile, HCPB Design Report 2015. Internal_Deliverable_BB-1.2.1-T002, (EUROfusion document, Working Package of Safety and Environment).
[11] J.C. Rivas, Development of AINA code for the study of loss of plasma control events in ITER and DEMO, and contribution to the systems study of DEMO, PhD thesis, Universitat Politècnica de Catalunya, 2016.

[12] ITER-Organization, GSSR Volume 11 - Models and Codes, XI (2001).

[13] N.A. Uckan, S. Putvinski, J. Wesley, H.-W. Bartels, T. Honda, T. Amano, D. Boucher, N. Fujisawa, D. Post, M. Rosenbluth, Iter physics-safety interface: models and assessments, (CONF-9606116--81). United States (1996). https://inis.iaea.org/Search/search.aspx?orig_q=RN:28020445

[14] Y. Yamamura, J. Bohdansky, Few collisions approach for threshold sputtering, Vacuum. 35 (1985) 561-571. https://doi.org/10.1016/0042-207X(85)90316-1

[15] W.D. Wilson, L.G. Haggmark, J.P. Biersack, Calculations of nuclear stopping, ranges, and straggling in the lowenergy region, Physical Review B. 15 (1977) 2458-2468. https://doi.org/10.1103/PhysRevB.15.2458

[16] C. García-Rosales, W. Eckstein, J. Roth, Revised formulae for sputtering data, Journal of Nuclear Materials. $218 \quad$ (1995) 8-17. https://doi.org/10.1016/00223115(94)00376-9

[17] R. Behrisch, G. Federici, A. Kukushkin, D. Reiter, Material erosion at the vessel walls of future fusion devices, Journal of Nuclear Materials. 313-316 (2003) 388-392.https://doi.org/10.1016/S0022-3115(02)01580-5

[18] F. Militello, W. Fundamenski, Multi-machine comparison of drift fluid dimensionless parameters, Plasma Physics and Controlled Fusion. 53 (2011). https://dx.doi.org/10.1088/0741-3335/53/9/095002

[19] P. Galli, A. Cherubini, R. De Angelis, F. De Luca, M. Erba, R. Giannella, G. Gorini, A. Jacchia, H. Jäckel, P. Mnatica, V. Parail, L. Porte, A. Taroni, Transient heat transport studies using laser ablated impurity injection in JET, Nuclear Fusion. (1998).

[20] W. Horton, W. Rowan, Impurity transport studies in the Texas Experimental Tokamak (TEXT), Physics of Plasmas. 1 (1994) 901-908. https://doi.org/10.1063/1.870749

[21] K.W. Gentle, W.L. Rowan, R. V. Bravenec, G. Cima, T.P. Crowley, H. Gasquet, G.A. Hallock, J. Heard, A. Ouroua, P.E. Phillips, D.W. Ross, P.M. Schoch, C. Watts, Strong nonlocal effects in a Tokamak perturbative transport experiment, Physical Review Letters. 74 (1995) 36203623. https://doi.org/10.1103/PhysRevLett.74.3620

[22] T. Honda, H.-W. Bartels, N.A. Uckan, Y. Seki, T. Okazaki, Development of Time Dependent Safety Analysis Code for Plasma Anomaly Events in Fusion Reactors, Journal of Nuclear Science and Technology. 34 (1997) 229-239. https://oi.org/10.1080/18811248.1997.9733655

[23] R. Wenninger, DEMO1 Reference Design - 2015 April ("EU DEMO1 2015"), 2MDKFH. (EUROfusion document, Working Package of Safety and Environment).

[24] F.A. Hernández, Thermo-hydraulic analyses of HCPB BB segment, EFDA_D_2HF7KU_v1.1, (EUROfusion document, Working Package of Safety and Environment).

[25] T. Pinna, Selection of reference accident scenarios for the DEMO plant. 2MA6TU_v1_0, (EUROfusion document, Working Package of Safety and Environment). 